\title{
Time dependencies in the occurrences of epileptic seizures
}

\author{
Leonidas D. Iasemidis ${ }^{a}$, Larry D. Olson ${ }^{b}$, Robert S. Savit ${ }^{c}$, J. Chris Sackellares ${ }^{b}{ }^{\text {,* }}$ \\ ${ }^{a}$ Department of Electrical Engineering, Bioengineering Program, Department of Neurology. Epilepsy Program, University of Michigan, \\ Ann Arbor, MI 48109-2122, USA: ${ }^{h}$ Department of Neurology, University of Michigan, Ann Arbor, MI 48109-0316. USA; \\ 'Physics Depurtment, University of Michigan, Ann Arbor. MI 48109, USA
}

(Received 6 October 1993; accepted 8 October 1993)

\begin{abstract}
A new method of analysis, developed within the framework of nonlinear dynamics, is applied to patient recorded time series of the occurrence of epileptic seizures. These data exhibit broad band spectra and generally have no obvious structure. The goal is to detect hidden internal dependencies in the data without making any restrictive assumptions, such as linearity, about the structure of the underlying system. The basis of our approach is a conditional probabilistic analysis in a phase space reconstructed from the original data. The data, recorded from patients with intractable epilepsy over a period of 1-3 years, consist of the times of occurrences of hundreds of partial complex seizures. Although the epileptic events appear to occur independently, we show that the epileptic process is not consistent with the rules of a homogeneous Poisson process or generally with a random (IID) process. More specifically, our analysis reveals dependencies of the occurrence of seizures on the occurrence of preceding seizures. These dependencies can be detected in the interseizure interval data sets as well as in the rate of seizures per time period. We modeled patient's inaccuracy in recording seizure events by the addition of uniform white noise and found that the detected dependencies are persistent after addition of noise with standard deviation as great as $1 / 3$ of the standard deviation of the original data set. A linear autoregressive analysis fails to capture these dependencies or produces spurious ones in most of the cases.
\end{abstract}

Key words: Partial epilepsy; Seizure recurrences; Nonlinear dynamics

\section{Introduction}

In some patients, seizures appear to occur unpredictably, with no discernable patterns. In others, seizures appear to occur in cycles. In some cases, the cycling patterns have been attributed to other biological rhythms such as the menstrual cycle. However, cycling has also been observed in

\footnotetext{
"Corresponding author.
}

males [1]. Clustering patterns, where one seizure appears to increase or decrease the likelihood of subsequent seizures, are a common clinical experience and have been reported in the literature $[2,3,15]$. In an article on seizure recurrences [23], Milton and co-workers concluded that the proportion of patients with seizure cycles and/or clusters is quite small with respect to the general population of patients. Using conventional statistical tests and assuming that the mean of the seizure 
rate is constant, they found that for most of the patients with multiple seizures, the pattern of seizure recurrence is random and follows a homogeneous Poisson distribution in $50 \%$ of the cases. In the remaining cases, seizures appeared to be randomly distributed but not according to a Poisson distribution. However, in that study, the number of seizures per patient was small and the rate of seizures they analyzed was low (on the average 18 seizures per patient and 1 seizure per 10 days). Moreover, the methods employed were not sensitive to many potential nonlinear dependencies in the data.

In patients with intractable partial seizures, Balish and co-workers [1] found evidence that seizures did not occur randomly in most of the patients. In that study, they fit the rate of seizure occurrences to a quasi-likelihood regression model. Using this model, they showed deviations from a homogeneous Poisson process. These deviations from the Poisson process included linear time trends and clustering. In addition, they found cycling at near-monthly intervals in four of their 13 patients. The cycling effects were detected through the "external input to their semi-linear model (non-autonomous time-dependent linear system). However, time dependencies that are not consistent with their model (e.g., nonlinearities in the structure of the generating system and/or cycling produced within the system) could be missed or misrepresented.

In recent years, it has become clear that deterministic low-order nonlinear systems can have extremely complicated, even chaotic behavior $[6,21,26,31]$. It has also become clear that standard methods of time series analysis, such as linear transforms and parametric linear modeling, are not useful in general for discerning the structure of such nonlinear systems, and may, in fact, lead to the erroneous conclusion that most of the signal is just random noise [29]. Consequently, it is important to develop and apply new methods for the analysis of the output of such systems [24]. Recently. we have applied methods from nonlinear dynamics to ECoG signals to investigate the transition from the preictal to the ictal to the postictal state $[14,16-18,28]$. These studies support the hypothesis that the occurrence of a seizure has, at least in part, a deterministic basis and is not merely a random process.

In this study, we analyze long-term seizure patterns in five adults with refractory complex partial seizures. Seizures diaries are used to generate two data sets per patient. The first data set is a time series in which each point represents the time interval between consecutive seizures. The sccond data set is a time series of the rate of seizure occurrence within consecutive prespecified time intervals. For statistical comparisons, each data set is shuffled to eliminate time dependencies. Thus, measures of time dependencies in the original data sets are compared with the measures in the shuffled (random) data sets. Because previous investigators $[1,23]$ have emphasized the Poisson distribution, we also compare the original data sets to Poisson distributed data sets with the same mean as the original data sets.

For the analysis of each data series, we employ a very recent method, developed within the framework of nonlinear dynamics, for detection of time dependencies in time series that are not resolved by conventional signal processing techniques $[14,28]$. This method has proven capable of detecting nonlinear dependencies and underlying structure in finite data series without the need for assumption of a particular model. The precise nature of the system that produces these dependencies cannot be determined from the estimated values of the indices of dependence alone. However, these indices do give information about which time lags are most important in the deterministic structure of the process. The application of the method to epileptic seizure data promises to shed light on the question of determinism of the occurrences of partial epileptic seizures.

In addition to the nonlinear analysis of our data sets, we report two other calculations. First, in order to measure the effect of possible inaccuracies in the reporting of seizures by patients, in each original data set a considerable amount of noise is added on the reported time of seizure occurrence and the estimated dependencies from the noisefree sets are compared to the ones from the noisy data sets. Finally, to facilitate comparisons with standard methods, we perform a linear autoregressive analysis on our data sets and we compare these results to those of our nonlinear analysis. 


\section{Methods}

\subsection{Patient selection}

Seizure diaries from five adult patients with partial seizures were analyzed. These patients were selected because they had kept detailed records over 1-3-year periods in which the date and time of each seizure was recorded. This provides data sets with a sufficient number of points to characterize the dynamical properties with the techniques employed. In each case, long-term EEG closed-circuit monitoring or ambulatory EEG cassette recordings confirmed that the patient was able to accurately report his or her seizure. The total number of recorded seizures is between 650 and 1200 per patient (see Table 1).

\subsection{Generation of data sets}

For each original series $s(n)$ of the occurrences of the epileptic seizures we create two new data series per patient. The first time series, denoted by $x(n)$, consists of the length of the interseizure intervals as a function of time. The second time series, denoted by $y(n)$ (rate of seizures over time), consists of the number of seizures that occur within a specific time interval (usually 1 day to 1 week) as a function of time. To determine the significance of the time dependence measures obtained from the patient data sets, each data set is shuffled to eliminate possible dependencies. Values of the proposed indices of dependence ( $\delta$ 's) estimated from the original time series are then compared to the ones from the randomized series and their significance is thereby determined* ${ }^{*}$.

To allow comparison with previous works, Poisson distributed data sets $\hat{s}(n)$ are also generated so as to have the same mean value as each patient's original data set $s(n)$. The corresponding data sets to $x(n)$ and $y(n)$, denoted by $\hat{x}(n)$ and $\hat{y}(n)$, are then generated. The statistical significance of the estimated dependence indices $(\delta$ 's) from the $\hat{x}(n)$ and $\hat{y}(n)$ data sets is determined through the shuffling process as described above.
Table 1

Statistical characteristics for all patients' $x(n)$, interseizure intervals, and $y(n)$, seizure rate per $T$ days, where $(x, y)$ are mean values, $\left(\sigma_{x}, \sigma_{y}\right)$ are standard deviations and $\left(N_{x}, N_{y}\right)$ are total number of events

\begin{tabular}{lccccc}
\hline Patient & I & II & III & IV & V \\
\hline Gender & M & F & F & F & F \\
$N_{x}$ & 921 & 776 & 668 & 1147 & 1007 \\
$x$ (days) & 0.61 & 2.93 & 1.96 & 3.90 & 0.92 \\
$\sigma_{x}$ (days) & 1.04 & 3.75 & 5.52 & 7.75 & 1.24 \\
$N_{y}$ & 564 & 567 & 657 & 696 & 924 \\
$T$ (days) & 1 & 4 & 2 & 7 & 1 \\
$y$ (\# seizures/day) & 1.63 & 1.37 & 1.90 & 1.81 & 1.10 \\
$\sigma_{y}$ (\# seizures/day) & 2.10 & 1.80 & 5.11 & 2.52 & 1.28 \\
\hline
\end{tabular}

Finally, uniform white noise $N(n)$ is also added to each data point $s(n)$ to compensate for possible inaccuracies on the recorded times of the seizures by the patients. We repeat the search for time dependencies in the thus resulted noisy data sets $x_{N}(n)$ and $y_{N}(n)$.

\subsection{Review of the proposed method of analysis}

Here we give a brief review of the proposed method of analysis for the time series of seizures (further details can be found in Savit and Green [28]). The initial step is to embed the one dimensional data in a higher dimensional space. To this end, one defines a $p$-dimensional vector $X_{p}$, whose elements are taken from the original time series with a time lag $\tau$ (method of delays $[32,34]$ ). That is, given a time series $x(n)$, where $n=1, \ldots, \mathrm{N}$ and $\mathrm{N}$ is the total number of data points available, vectors $X_{p}(i)$ are constructed in a $p$-dimensional phase space by:

$$
X_{p}(i)=\{x(i-(k-I) \times \tau) ; \mathrm{k}=1, \ldots, \mathrm{p}\}
$$

with $\tau$ being the time lag used for the embedding and $i=p \times \tau, \ldots, N$. Now consider two of these vectors $X_{p}(i)$ and $X_{p}(j)$ constructed from the time series. The probability $P$ that the two vectors

\footnotetext{
* Logically, comparisons with shuffled data sets amounts to a test against the null hypothesis that the sequence of numbers being tested is random in the sense of IID (independent and identically distributed). The shuffling procedure which produces the null set is an application of the statistical technique of bootstrapping [9].
} 
have all their cartesian components within a distance $\varepsilon$ of each other respectively can be approximated by $[12,13]$

$$
\left.C_{p}(i)=\frac{1}{(N-p \times \tau+1)^{2}} \sum_{i=p<\tau}^{v} \sum_{p=\tau}^{N} \theta_{(i,-}\left\|\mathrm{X}_{\mathrm{p}}(\mathrm{i})-\mathrm{X}_{\mathrm{p}}(\mathrm{j})\right\|\right)(2)
$$

where $\Theta$ equals 1 for positive and 0 for negative arguments and \|\| represents the max norm, that is the maximum of the distances between the components of any two vectors $X_{p}(i)$ and $X_{p}(j) . \mathrm{C}_{\mathrm{p}}(\varepsilon)$ is called the correlation integral.

If $x(n)$ is an IID (independent and identically distributed) random process then $C_{p}(\varepsilon) \cong C_{1}{ }^{p}(\varepsilon)$ [28]. A test against the null hypothesis of IID can be constructed by defining

$$
\delta_{1}(\varepsilon)=\frac{C_{2}(\varepsilon)-C_{1}{ }^{2}(\varepsilon)}{C_{2}(\varepsilon)}=1-\frac{C_{1}{ }^{2}(\varepsilon)}{C_{2}(\varepsilon)}
$$

so that $\delta_{1}(\varepsilon) \cong 0$ for IID. The extent to which $\delta_{1} \neq 0$ is an indication of some structural dependence on the first lag in the lime series.

Similarly, we can define, for $k=2, \ldots, p-1$

$$
\delta_{k} \doteq 1-\frac{C_{k-1}(\varepsilon) \times C_{k+1}(\varepsilon)}{C_{k}^{2}(\varepsilon)}
$$

The extent to which $\delta_{k} \neq 0$ is a measure of existence of dependence in the time series on the $k$ th lag, given that we have used the information of the previous $(k-1)$ lags. If there is no additional dependence on the $k$ th lag, aside from that induced by the 1 st, 2 nd, $, \ldots,(k-1)$ th lags, then $\delta_{k}=0$. In particular, for an infinitely long IID series, $\delta_{k}=0$ for all $k$ 's.

Values of $\partial_{k}(\varepsilon)$ 's are calculated after choosing a tolerance $\varepsilon$. Typically $\varepsilon$ is chosen as some moderate fraction of the standard deviation of the data set $[14,28]$. Non-zero values, that are also statistically significant (see the 'statistical significance' section), indicate which time lags contain deterministic information at a given value of $\delta$ in the sense described above.

Like all statistical methods, the calculation of the $\delta_{k}(\varepsilon)$ is subject to a number of empirical caveats. The simplest interpretation of the $\delta_{k}(\varepsilon)$ 's follows from application to a stationary time series. In addition, as $k$ increases, so do the data requirements. For example, with a time series of about 500 points and an $\varepsilon$ of $1 / 2$ the standard deviation of the values of the data set. $\delta_{k}$ 's for $k \geqslant 5$ are generally not statistically significant. The power of the method lies in the fact that nonlinear effects in the time series that are missed by standard statistical techniques are often uncovered, and that the method uses the data in a very efficient way (more details can be found in Savit and Green [28] and Wu et al. [35]).

\subsection{Linear autoregressive modeling}

The AR model is a popular model for modeling linear systems. A $p$-th order linear autoregressive (AR) model is given by the equation

$$
s(n)=\sum_{i}^{n} a_{i} \times s(n-i)
$$

The estimation of the prediction coefficients $a_{i}$ 's is based on the minimization of the prediction error. The coefficients $a_{i}$ reflect not only the explicit dependencies in the series but the implicit ones too. As a result, the estimated values of the $a_{i}$ 's depend on the order of the AR model used. The reflection coefficients $k$,s, quantities that relate to the $a_{i}$ 's, do not depend on the order of the AR model [27]. If there exists a low order linear dependence in the data, it will affect only the corresponding low order $k_{i}$ reflection coefficient. Therefore, the reflection coefficients $k_{i}$ 's were estimated in this study. Burg's algorithm is used for the estimation of the $a$ 's and $k$ 's $[5,20,22]$. The limitations of the AR modeling of nonlinear systems are demonstrated by a simulation example (see the 'Results' section).

\subsection{Statistical hypothesis testing}

The null hypothesis is that the time series under investigation is IID. If the null hypothesis is true, then the estimated indices of dependence $\delta_{k}$ 's for the original data should take values very close to zero. But for any finite data set the $\dot{\delta}_{k}$ 's will not be exactly zero. To assess their significance we use the method of bootstrapping, and repeatedly shuffle the original time series to produce a set of new time scrics all of which have the same overall probability distribution of values as the original time series, but which are IID $[8,9]$. The value of $\delta_{k}$ for the original time series is compared with the distribution of values of $\delta_{k}$ for the set of rando- 
mized series. If the original $\delta_{k}$ is significantly larger than the mean $\vec{\delta}_{k}$ for the randomized set, then we say that this $\delta_{k}$ is statistically significant. Typically, the 'set of $\delta_{k}$ 's from shuffled series form a bell-like distribution of values. Along the lines of the $t$-test [33], we define as a measure of the statistical significance of the original $\delta_{k}$, the absolute difference of $\delta_{k}$ from the mean value $\bar{\delta}_{k}$ normalized by the standard deviation $\sigma$ of the $\delta_{k}$ 's of the shuffled series. Then, the statistical significance level $(S S)$ of a $\delta_{k}$ is given by

$$
S S=\operatorname{erf}\left(\frac{\left|\delta_{k}-\bar{\delta}_{k}\right|}{\sigma} / \sqrt{2}\right)
$$

where erf is the error function, well tabulated in statistics literature. As a result, the null hypothesis will be rejected at the $L \%$ confidence level, and $\delta_{k}$ will be considered statistically significant at the $\mathrm{L} \%$ level, if $\mathrm{SS}$ is greater than $L$ (we will use a $\mathrm{L}=95$ confidence level, unless otherwise noted). If the value of the original $\delta_{k}$ differs from $\bar{\delta}_{k}$ by more than 2.5 times the standard deviation of the distribution of the values of $\delta_{k}$ from the shuffled series, we may be confident at the $95 \%$ level that this $\delta_{k}$ is significant. This statistical treatment is adequate for our semi-quantitative purposes here. However, because the $\delta_{k}$ 's of IID data do not necessarily have a precisely normal distribution, the estimates of statistical significance used may not be precise (see Wu et al. [35] for details).

\section{Results}

\subsection{A simulation example: the logistic map}

For pedagogical purposes, we review here the application of the method to the logistic map, a simple chaotic map. We shall see that our method correctly indicates structure in this system even when linear methods fail. The equation of the logistic map

$$
s(n)=a \times s(n-1) \times[1-s(n-1)]
$$

is used with $a=4$, so that the system is in the chaotic regime $[7,10,19], s(1)=0.1$ and $n=2, \ldots, N$, where $N=1000$. It is obvious that, even in the chaotic regime, a one-step time dependence exists

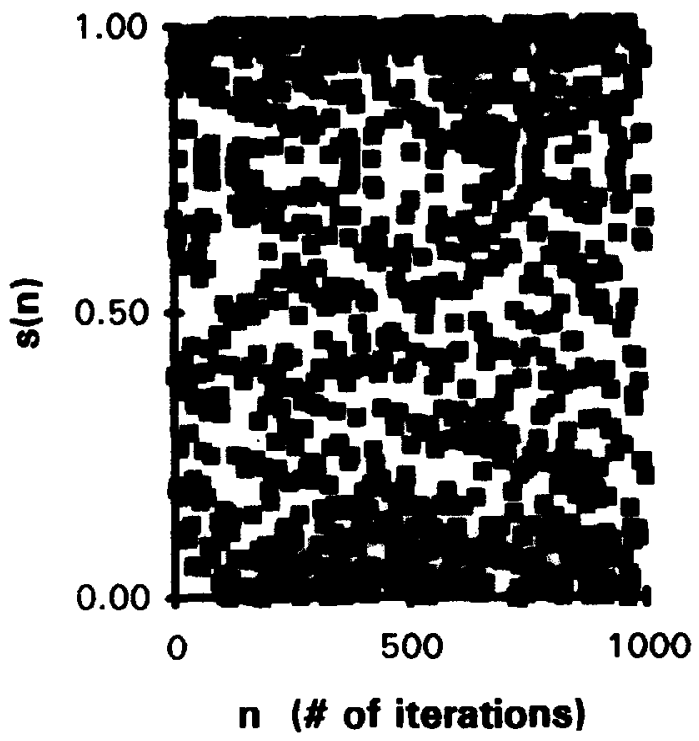

Fig. 1. Logistic map data $s(n)$ from the chaotic regime $(a=4$, $\left.\mathrm{s}(1)=0.1, \mathrm{~N}=1000, \sigma_{\mathrm{s}}=0.35, \overline{\mathrm{s}}=0.51\right)$. The temporal pattern appears to be randon even though it is generated by a purely deterministic model.

in $s(n)$, created by the one-step explicit time dependence in the system that produces $s(n)$ (see Eq. 7). The raw data $s(n)$ are given in Fig. 1 for 1000 iterations of the map. It is clear from this figure that there is no obvious temporal structure in the series $s(n)$.

The $\delta(\varepsilon)$ 's for $s(n)$ arc estimated using $\tau=1$ (to capture the highest frequency present in the data) and $\delta_{k}$ for $k=1,2,3$ are shown in Fig. 2(a). From Fig. 2(a) one can see that the absolute value of $\delta_{1}$ is about an order of magnitude larger than either $\delta_{2}$ or $\delta_{3}$, over the whole range of $\varepsilon$. This result strongly suggests that a one-step time dependence exists in the data (but see Savit and Green [28] for a technical caveat). To check for statistical significance, 100 shuffled data sets were produced from the original data set $s(n)$ (for the production of the first shuffled data set, the original data were shuffled 15 times. Subsequent (15 times) reshuffling of each generated shuffled data is used to produce a total of 100 shuffled data sets in addition to the original data set). The $\delta(\varepsilon)$ 's of the shuffled data sets are estimated using the same $\tau$. The $\delta_{1}(\varepsilon)$ of both the original and the shuffled data sets is plotted in Fig. 2(b). From Fig. 2(b), it is 

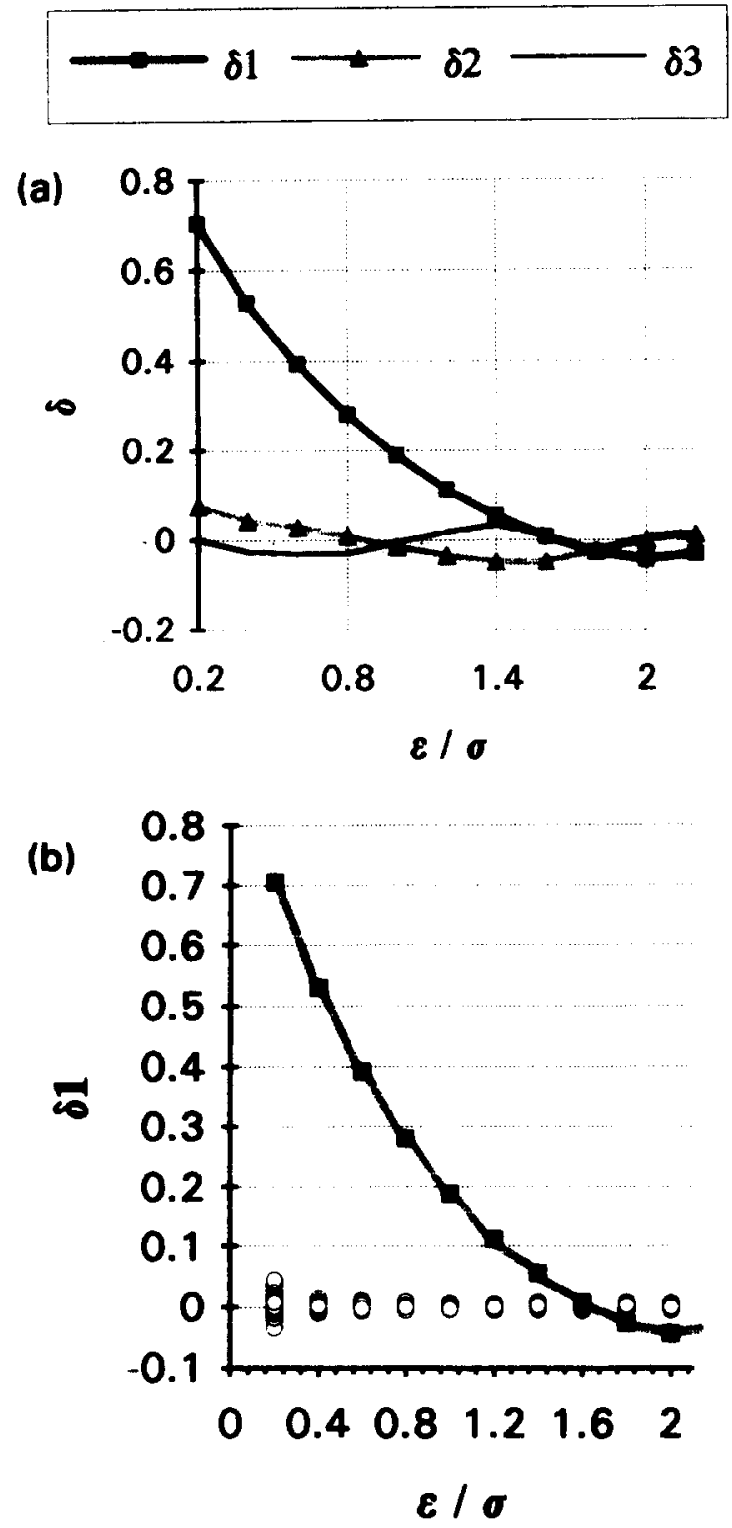

Fig. 2. (a) $\delta_{1}, \delta_{2}, \delta_{3}$ versus $\varepsilon$ for the logistic data $s(n)$. As expected, $\delta_{1}$ is large whereas $\delta_{2}, \delta_{3}$ are near 0 . (b) $\delta_{1}$ versus $\delta$ for the logistic data $s(n)$ (solid line) and for the shuffled versions of $s(n)$ (100 scattered points at each value of $\varepsilon)$. The statistical significance of $\delta_{1}$ is thus established.

clear that $\delta_{1}$ is statistically significant, since for small values of $\varepsilon, \delta_{1}$ of the unshuffled data is more than 5 standard deviations from the mean $\bar{\delta}_{1}$ of the shuffled data sets. For large $\varepsilon$, the tolerance with which the data are being examined is sufficiently crude that none of the structure within the sequence is discernible, and so, at this level, the logistic data look random. Thus, for large $\varepsilon, \delta_{k}$ is not statistically significant. Finally we remark that if uniform white noise is added to the output of the logistic map data (up to $10 \mathrm{~dB}$ signal to noise ratio, that is, addition of noise having standard deviation equal to half of the standard deviation of the original data), $\delta_{1}$ is still much larger than the rest of the $\delta$ 's and is also statistically significant [28]. This suggests that the proposed indices of dependencies are quite robust, at least for systems as complex as the chaotic logistic map.

To compare the efficacy of this method with linear methods we attempt to fit the logistic map time series with a 3 rd order linear autoregressive model after subtraction of the mean value of the data from the data series. The shuffled data sets of $s(n)$ are also fitted with a 3 rd order AR model. The values of $k_{1}$ to $k_{3}$, from the original logistic data and shuffled versions of the data were estimated and $k_{1}$ and $k_{2}$ were not found to be statistically significant $\left(k_{1}=+0.02 ; \quad S S=42.93\right.$ and $k_{2}=+0.01 ; S S=15.12$ ). Moreover, $k_{3}$ is marginally statistically significant at confidence level $82.25 \%\left(k_{3}=-0.05 ; S S=82.25\right)$ and thus constitutes a spurious dependence, which is created by the AR modeling and does not exist in the original data. This is in disagreement with the explicit one-step dependence of the logistic equation. The prediction error, illustrated in Fig. 3, shows that almost no improvement in our predictability can be achieved by fitting the data with a 3 rd order AR model, and the prediction error of the model almost equals the standard deviation of the data. Moreover, any higher order AR will also fail to capture the one-step dependence, because, as explained above, the lower order $k$ 's maintain their values as we increase the order of the model and thus they remain insignificant. Therefore, it is clear that the statistical methods described in the Methods section can reveal nonlinear structures to which ordinary linear methods are insensitive.

\subsection{Interseizure intervals}

The interseizure interval data $x(n)$ are generated from the original data $s(n)$ by: 


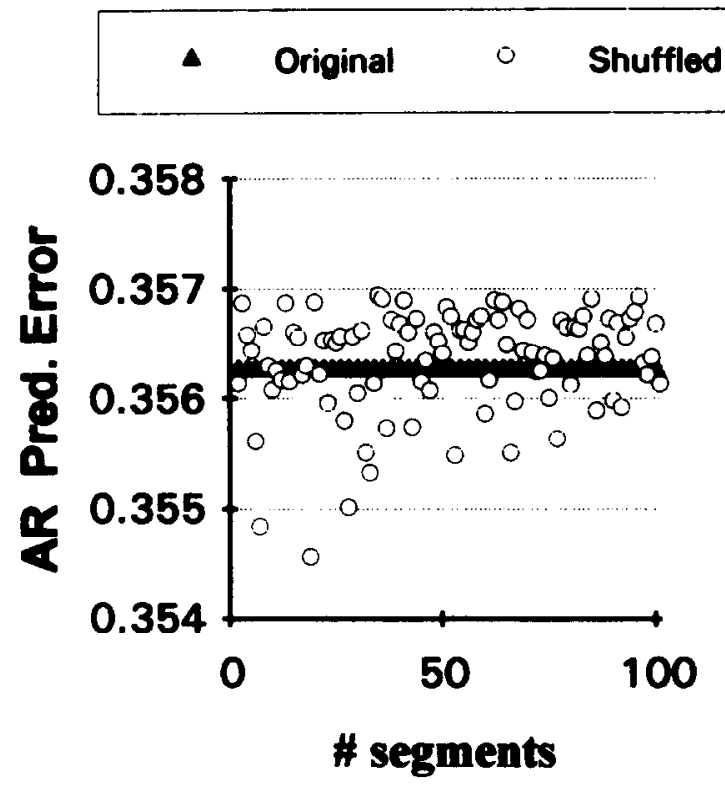

Fig. 3. Prediction error from the 3rd order AR fit to the logistic map (solid line) and to the shuffled versions of it (scattered points). The prediction error is statistically insignificant.

$$
x(n)=s(n)-s(\mathbf{n}-1)
$$

In Fig. 4(a) and 4(b), the interseizure intervals $x(n)$ from patient $\mathrm{I}$, and the interarrival intervals of a Poisson process created with the same mean value as $\hat{x}(n)$, are shown.

Since previous authors have discussed the possibility that seizure occurrences are Poisson distributed, we compare quantitatively the deviation of the data from the corresponding homogeneous Poisson process in terms of the estimated $\delta$ 's for both processes. In Table 2, we list the values of $\delta_{2}$ and their statistical significance (SS) vs. $\varepsilon$ for paticnt I, with $\tau=1$, for both of the above data sets $x(n)$ and $\hat{x}(n)$. We first cite the values of $\delta \mathrm{d}_{2}$, next their statistical significance.

The $\delta$ 's are calculated according to Eq. 4, for different values of the tolerance $\varepsilon$ ( $\varepsilon$ from $\sigma / 5$ to $2 \times \sigma$, where $\sigma$ is the standard deviation of the data under consideration). It is clear that the values of $\delta_{2}$ for the interseizure intervals in this patient are considerably larger than the ones for the Poisson interarrival intervals, over almost the entire $\varepsilon$ domain, which implies an overall dependence of each interseizure interval on the 2 nd previous
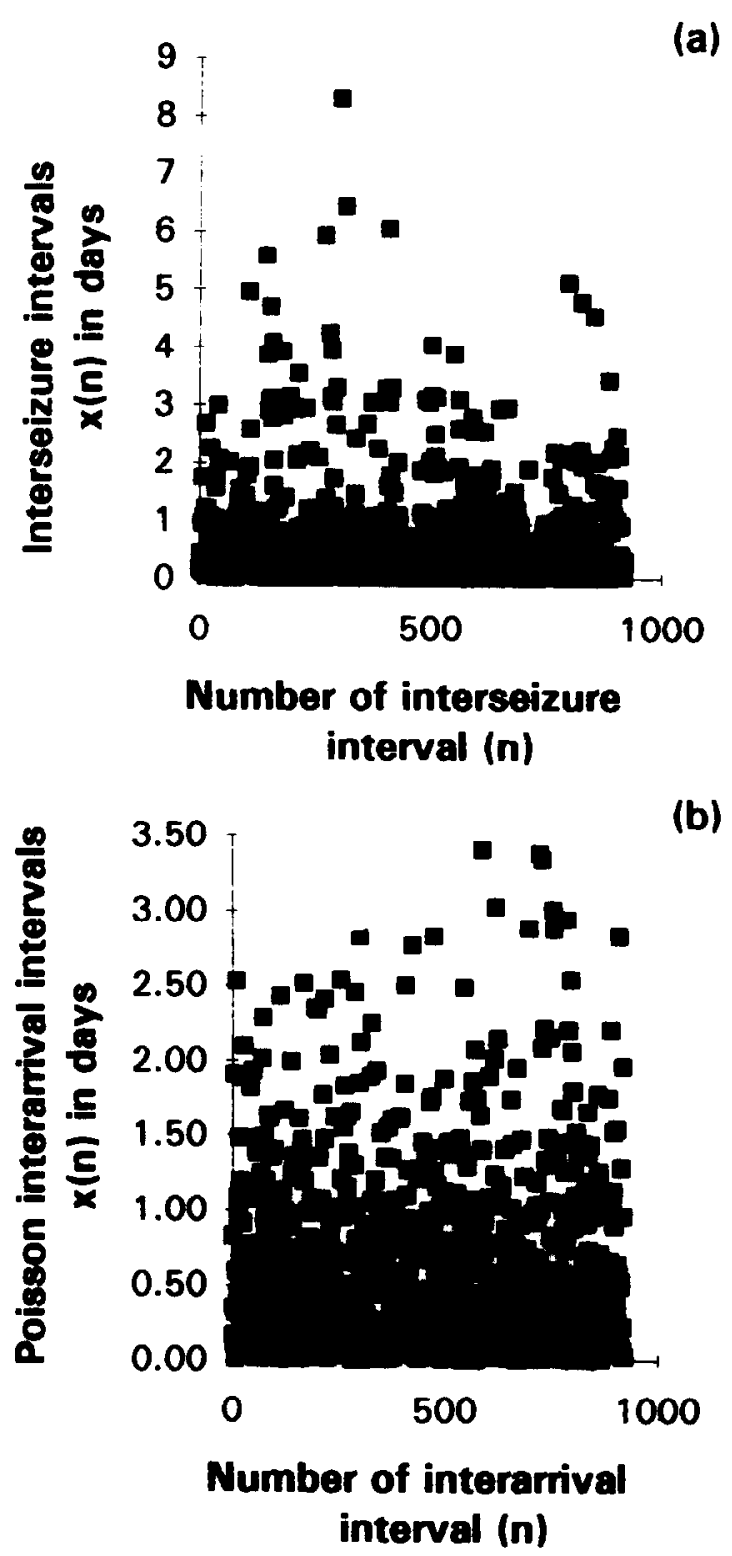

Fig. 4. For patient I: (a) Interseizure intervals $x(n)(\mathrm{N}=921$, $\overline{\mathbf{x}}=0.61$ days, $\sigma=1.04$ days). (b) Interarrival intervals from a homogeneous Poisson process $\hat{x}(n)(\mathrm{N}=921, \overline{\mathrm{x}}=0.61$ days, $\sigma=0.62$ days). Visual inspection of the above graphs reveals no discernible temporal pattern in either data set.

one in this patient. Larger dependencies, as indicated by larger values of the $\delta$ 's for $x(n)$ than for $\hat{x}(n)$ are found for every data set for all five patients. The $\delta$ 's estimated from Poisson distribu- 
Table 2

Value of $\delta_{2}$ and its statistical significance $S S$ as a function of $\delta / \sigma$ for the interseizure intervals $x(n)$ of patient I and the corresponding Poisson interarrival intervals $\hat{x}(n)$

\begin{tabular}{llc}
\hline $\begin{array}{l}\text { Normalized } \\
\text { tolerance } c / \sigma\end{array}$ & $x(n)\left(\delta_{2} ; S S\right)$ & $\dot{x}(n)\left(\delta_{2} ; S S\right)$ \\
\hline 0.2 & $(0.10 ; 99.99)$ & $(-0.01 ; 15.05)$ \\
0.4 & $(0.06 ; 99.85)$ & $(0.00 ; 52.23)$ \\
0.6 & $(0.05 ; 99.95)$ & $(0.00 ; 12.25)$ \\
0.8 & $(0.03 ; 92.85)$ & $(0.00 ; 5.00)$ \\
1.0 & $(0.02 ; 93.50)$ & $(0.00 ; 10.30)$ \\
1.2 & $(0.01 ; 96.60)$ & $(0.00 ; 31.15)$ \\
1.4 & $(0.01 ; 98.91)$ & $(0.00 ; 4.00)$ \\
1.6 & $(0.01 ; 98.95)$ & $(0.00 ; 3.00)$ \\
1.8 & $(0.01 ; 99.60)$ & $(0.00 ; 42.84)$ \\
2.0 & $(0.01 ; 98.93)$ & $(0.00 ; 51.41)$ \\
2.2 & $(0.01 ; 95.23)$ & $(0.00 ; 22.27)$ \\
\hline
\end{tabular}

tions should statistically be equal to zero (since the interarrival intervals in a Poisson process are IID $[11,25,30])$. And, as we see in Table 2, all values of $\delta$ 's from the Poisson data set are found to be zero to two significant figures.

In order to establish the statistical significance of the observed dependence against an IID hypothesis, new data sets are created from the original $x(n)$ by shuffling. The $\delta_{2}$, for both the raw and shuffled data sets from patient I, for $\tau=1$, is plotted as a function of $\varepsilon$ in Fig. 5(a). The $\delta_{2}$ with $\tau=1$, for both the raw and shuffled data sets of the homogeneous Poisson data $\hat{x}(n)$ for patient $I$, is plotted as a function of $\varepsilon$ in Fig. 5(b). The statistical significance of $\delta_{2}$ of the $x(n)$ and $\hat{x}(n)$ data from patient $\mathrm{I}$ is given for successive values of $\varepsilon$ in Table 2. From this Table, it is clear that $\delta_{2}(\varepsilon)$ of the original data set is statistically significant over a large region of $\varepsilon$. The insignificance of the $\delta_{2}$ of the corresponding Poisson data over the whole range of $\varepsilon$ is also seen.

Given the existence of these dependencies, it is natural to ask whether a simple linear AR process can describe the above series of interseizure intervals. To this end, we employ a 3rd order linear AR model and proceed as in the logistic map example. For patient I, the fitting of the AR to the $x(n)$ and their shuffled versions, results in $\left(k_{1}=-0.09\right.$; $S S=99.53), \quad\left(k_{2}=-0.06 ; \quad S S=95.23\right) \quad$ and
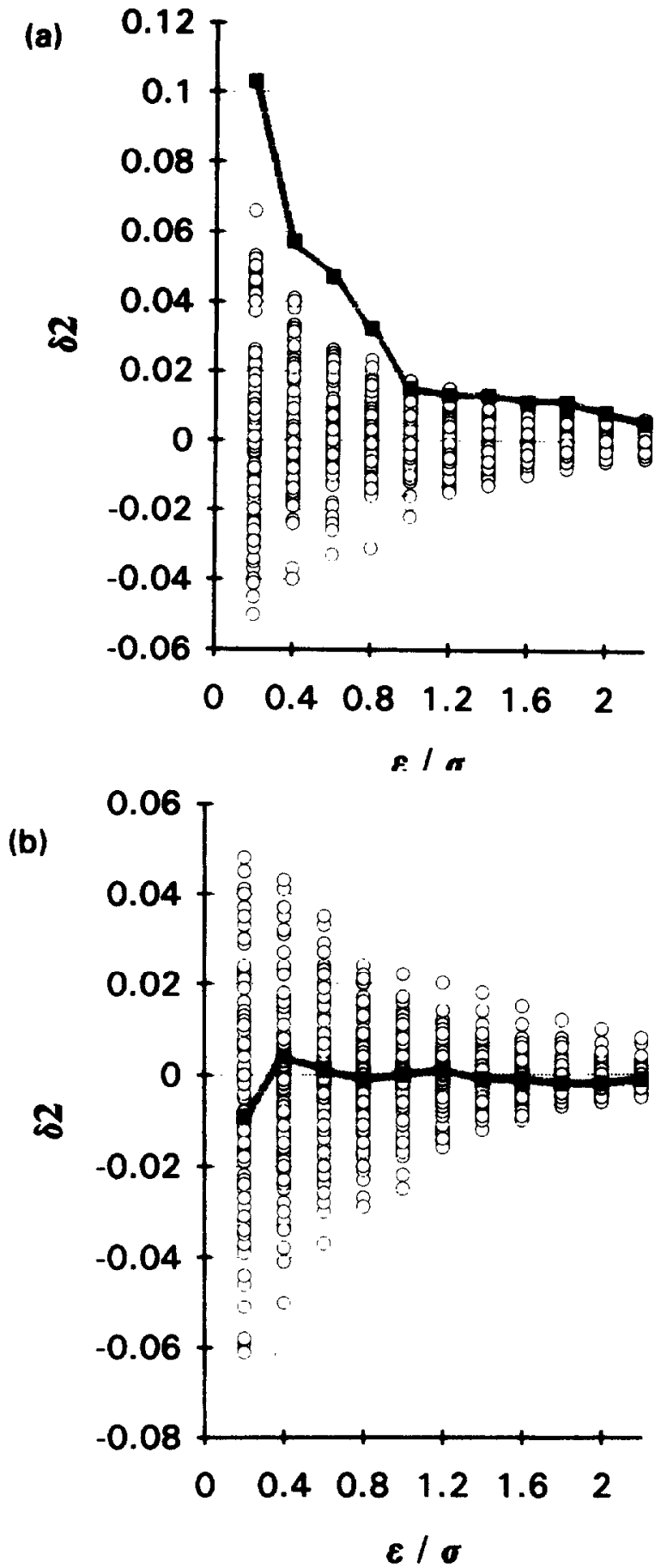

Fig. 5. (a) $\delta_{2}(\tau=1)$ as a function of $8 / \sigma$ for the interseizure intervals $x(n)$ for patient $I$ (solid line) and the shuffled versions of $x(n)$ (scattered points). (b) As in (a) but for the corresponding homogeneous Poisson intervals $\hat{x}(n)$ and its shuffled versions. The $\delta_{2}$ is statistically significant for the interseizure intervals but not at all for the Poisson interevent intervals. 
Table 3

The most statistically significant pairs $(\delta, \tau)$, at $\varepsilon=\sigma / 2$ and confidence level $99 \%$, for the interseizure interval data for all patients

\begin{tabular}{lllll}
\hline Patient I & Patient II & Patient III & Patient IV & Patient V \\
\hline$\left(\delta_{2}, \tau=1\right)$ & $\left(\delta_{1}, \tau=1\right)$ & $\left(\delta_{1}, \tau=1\right)$ & $\left(\delta_{1}, \tau=5\right)$ & $\left(\delta_{2}, \tau=25\right)$ \\
& $\left(\delta_{1}, \tau=3\right)$ & $\left(\delta_{2}, \tau=5\right)$ & $\left(\delta_{2}, \tau=3\right)$ & \\
& $\left(\delta_{1}, \tau=4\right)$ & $\left(\delta_{5}, \tau=2\right)$ & & \\
& $\left(\delta_{1}, \tau=5\right)$ & & & \\
\hline
\end{tabular}

$\left(k_{3}=-0.05 ; S S=87.50\right)$. We notice that only the first reflection coefficient of the AR is statistically significant at the $99 \%$ confidence level. The second coefficient $k_{2}$ is statistically significant at the $95 \%$ confidence level. This is to be compared with the insignificance of $\delta_{1}$ for this patient (see Table 3 ) and the strong significance of $\delta_{2}$ for a large range of $\varepsilon$ as it was described above. These divergent results call into question the ability of the AR model to fit these data. Application of the AR model to $x(n)$ data of the other patients gives similar results.

The failure of the AR to fit the data may be attributed to the existence of possible nonlinear relations between the various data samples. This interpretation is supported by the results of the AR analysis in the logistic map, where even a 3rd order AR could not detect the presence of a 1st order (one-step) nonlinear dependence. One could argue that the order of the AR model may be too small to capture any structures in the data. However, since there are statistically significant low order dependencies in our data that can be detected through the $\delta$ 's, a very high order AR is likely to only introduce spurious higher order dependencies.

Another drawback in the use of an AR occurs when missing data (i.e., no recording of the time of a seizure) exist in the middle of a record. This affects considerably the estimation process because of errors that are produced during the sequential estimation of the covariance matrices. However, a small number of missing data does not seem to present a serious problem in the estimation of $\delta$ 's because of the global nonsequential nature of the statistics. For example, one can compensate for the missing part of the data set by omitting any vector that contain missing data values in its components in the reconstruction of the phase space.

An attempt to investigate the persistence of the detected dependencies (i.e., in the case of inaccurate recording of the time of seizures) is made by the addition of white uniform noise $N(n)$ to $x(n)$ and subsequent estimation of the $\delta$ 's. When the standard deviation of the noise exceeds about $1 / 3$ of the standard deviation, $\sigma$, of the data $x(n)$, the dependencies in the data begin to be obscured (see Fig. 6). Longer data sets are necessary to reveal dependencies in a complex system with a signal to noise ratio much less than 1 (see Savil and Green [28] for more details).

Thus, the seizures of this patient do not seem to occur independently or according to a homogeneous Poisson process. Similar strong low order dependencies were observed in the interseizure interval data sets in four out of the five patients we analyzed (for example, see Fig. 7 for $\delta_{1}$ of patient III). For $\varepsilon=\sigma / 2$, and for $1 \leqslant k \leqslant 5$ and $1 \leqslant \tau \leqslant 5$, we have listed in Table 3 , the pairs $\left(\delta_{k}, \tau\right)$ which show statistical significance at the $99 \%$ confidence level for all patients. Patient $\mathrm{V}$ exhibited only a marginal $\delta_{2}$ at $\tau=1$, at small values of $\varepsilon$, but did show strong long-term dependencies for $\tau=25$.

\subsection{Rate of seizures data}

The rate of scizurcs, $y(n)$, is constructed from each original data set $s(n)$ and consists of the number of seizures that occurred within a specific period $T$ (i.e., period of 1 day up to 1 week). $T$ is selected such that the resulting data set, $y(n)$, produces enough vectors for a reasonable higher dimensional analysis and relatively small quantization error in the estimation of $\delta$ 's (very small $T$ produces low seizure rates over time and one would need a very small $\varepsilon$ to discern any structure in the data; very large $T$ smooths the seizure rate and obscures any structure in the data). A low multiple of the mean of the seizure rate constitutes a reasonable choice for $T$. The statistically significant pairs $(\delta, \tau)$ for all patients' rate of seizures are given in Table 4.

The rate of seizure over time data for patient I is given in Fig. 8(a) for $T=1$ day. In Fig. 8(b), the interarrival intervals $\hat{x}(n)$ of a Poisson process 
created with the same mean value as $y(n)$ are shown. Only $\delta_{1}$ is found to be statistically significant for this patient, which means that the number of seizures of patient I that occur in one day de-

(a)

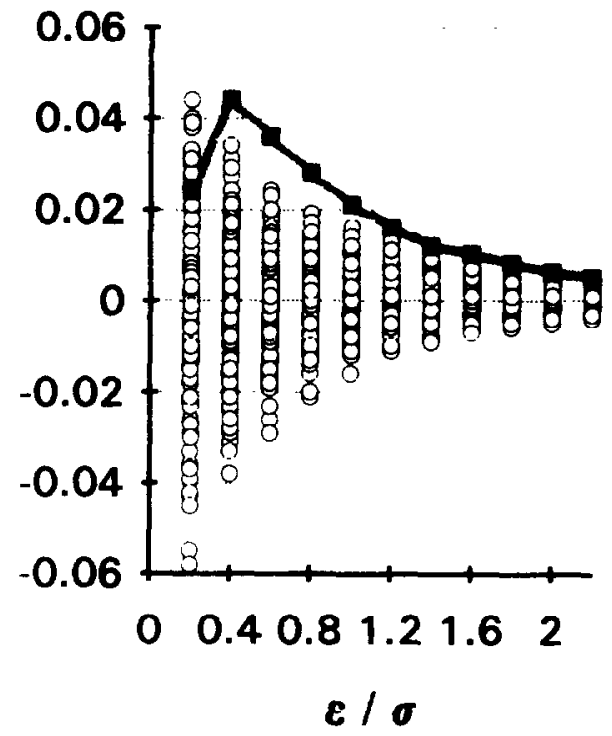

(b)

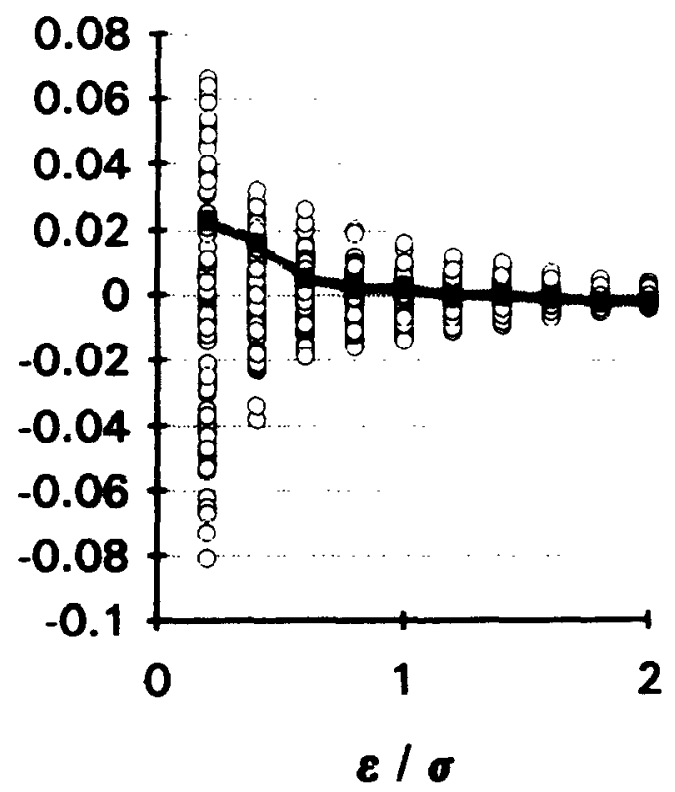

Fig. 6. $\delta_{2}(\tau=1)$ as a function of $\varepsilon / \sigma$, for the interseizure intervals $x(n)$ of patient $I$ (solid line) and the shuffled versions of $x(n)$ (scattered points). when uniform white noise is added to the times of scizure occurrences $s(n)$ with standard deviation (a) $\sigma_{N}=0.3 \times \sigma$, (b) $\sigma_{N}=1.0 \times \sigma$. The $\delta_{2}$ is statistically significant with addition of noise up to $\sigma_{N}=0.3 \times \sigma= \pm 8 \mathrm{~h}$.

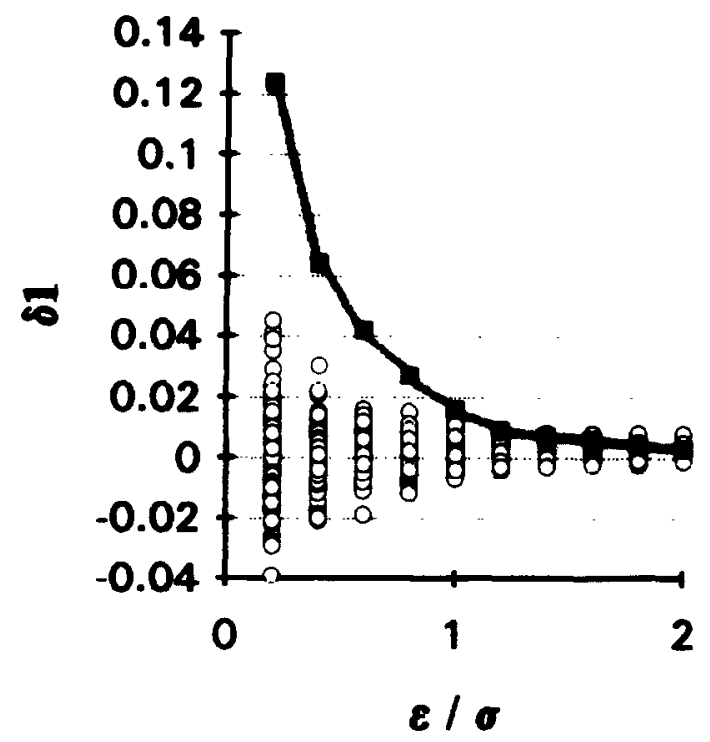

Fig. 7. $\dot{\delta}_{1}(\tau=1)$ as a function of $\varepsilon / \sigma$, with $\sigma=5.52$ days, for the interseizure intervals $x(n)$ of patient III (solid line) and the shuffled versions of $x(n)$ (scattered points). This dependence is more statistically significant than the one in patient I (see Fig. 5).

pends on the number of seizures that occurs during the previous day. The estimated $\delta_{1}(\varepsilon)$ of $y(n)$ from patient $\mathrm{I}(T=1$ day) is given in Fig. 9(a) and the corresponding $\delta_{1}(\varepsilon)$ for Poisson data in Fig. 9(b) (the identical value of $\delta_{1}$ for sequential values of $\varepsilon$ in these graphs is a simple discrete binning effect). Since the mean number of seizures per day for this patient is $\bar{y}=1.63$, this result suggests the existence of a relationship over approximately $3(\sim 2 \times \bar{y})$ successive seizures. It is noteworthy that $\left(\delta_{2}, \tau=1\right)$ was found to be the most significant pair from the analysis of the interseizure intervals $x(n)$ for this patient (see Fig. 5, Table 3), which implicd a dependence of one interseizure interval on the previous second interval, that is

Table 4

The most statistically significant pairs $(\delta, \tau)$, at $\varepsilon=\sigma / 2$ and confidence level $99 \%$, for the rate of seizures data (number of seizures per $T$ days) of Table 1 for all patients

\begin{tabular}{lcccc}
\hline Patient I & Patient II & Patient III & Patient IV & Patient V \\
\hline$\left(\delta_{1}, \tau=1\right)$ & $\left(\delta_{1}, \tau=1\right)$ & $\left(\delta_{1}, \tau=1\right) \quad\left(\delta_{1}, \tau=1\right)$ & $\left(\delta_{2}, \tau=1:\right.$ \\
$\left(\delta_{2}, \tau=1\right)$ & $\left(\delta_{2}, \tau=1\right)$ & $\left(\delta_{3}, \tau=1\right)$ & & margmal $)$ \\
& & $\left(\delta_{3}, \tau=1\right)$ & &
\end{tabular}


consistent with an interrelation over three successive seizures. As expected, no statistically significant $\delta_{k}$ is found by the analysis of $y(n)$ of the corresponding homogeneous Poisson process.

For patient II, strong dependencies are found
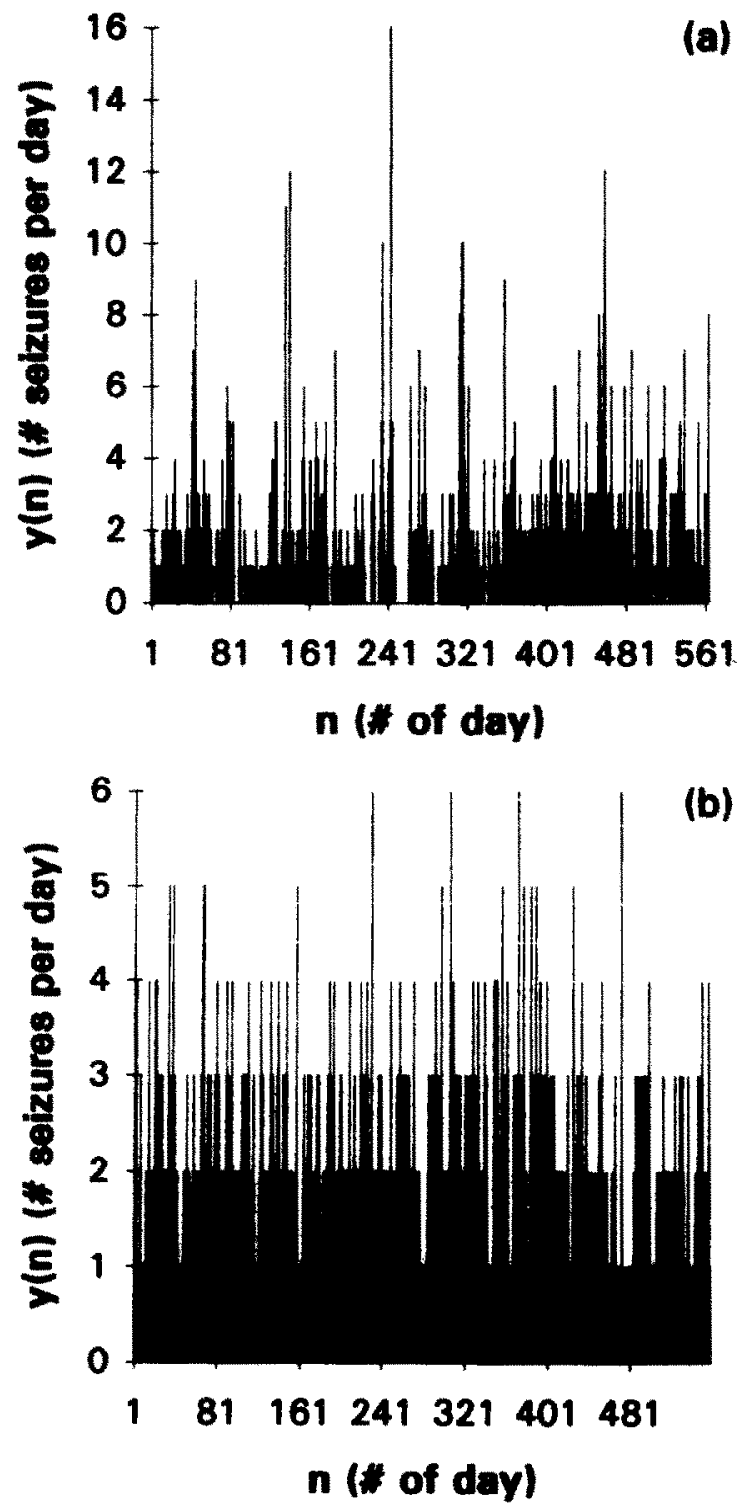

Fig. 8. For patient I: (a) Number of seizures per day over time $(y(n))\left(\mathrm{N}=559, \sigma_{y}=2.10, \overline{\mathrm{y}}=1.63\right)$. (b) Number of seizutes per day over time for the corresponding Poisson process $\hat{y}(n)$ $\left(\mathrm{N}=559, \sigma_{y}=1.32, \bar{y}=1.64\right)$. Visual inspection of the above graphs reveals no discernible temporal pattern in either data set. (a)

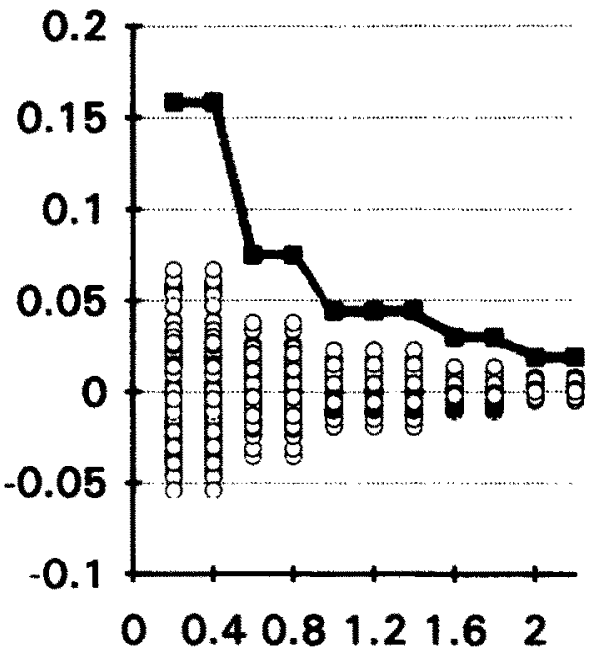

(b)

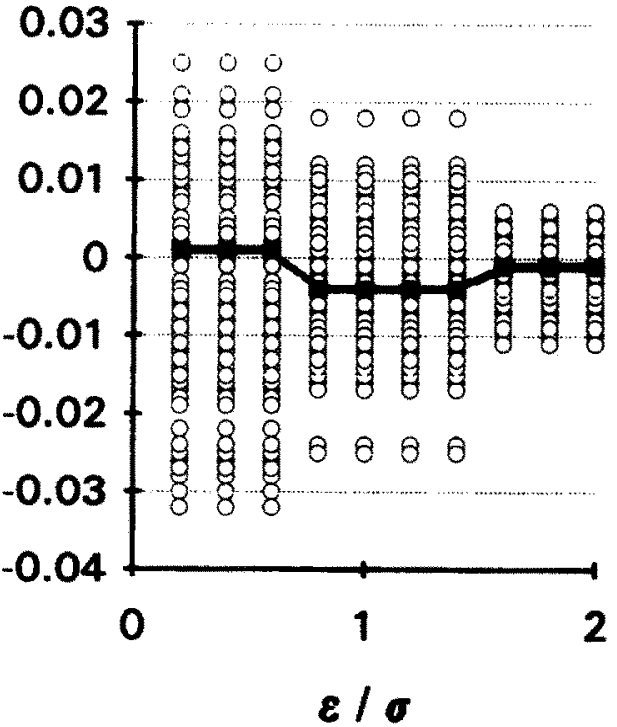

Fig. 9. (a) $\delta_{1}$ for the rate of seizures data $y(n)(T=1$ day $)$ as a function of $\varepsilon / \sigma$ for patient I (solid line) and the shuffled versions of $y(n)$ (scattered points). (b) As in (a) but for the corresponding homogeneous Poisson rate data. The $\delta_{1}$ is clearly statistically significant.

in the $y(n)$ data for one lag with $T=1$ day, for three lags with $T=2$ days, and for one and two lags with $T=4$ days. These results are also in qualitative agreement with the large scale dependencies found among the interseizure intervals for this patient (see Table 3).

For the $y(n)$ data from patient III, the most significant pair is found to be the $\left(\delta_{1}, \tau=1\right)$ using $T=2$ days (see Fig. 10). Noting that in this patient 


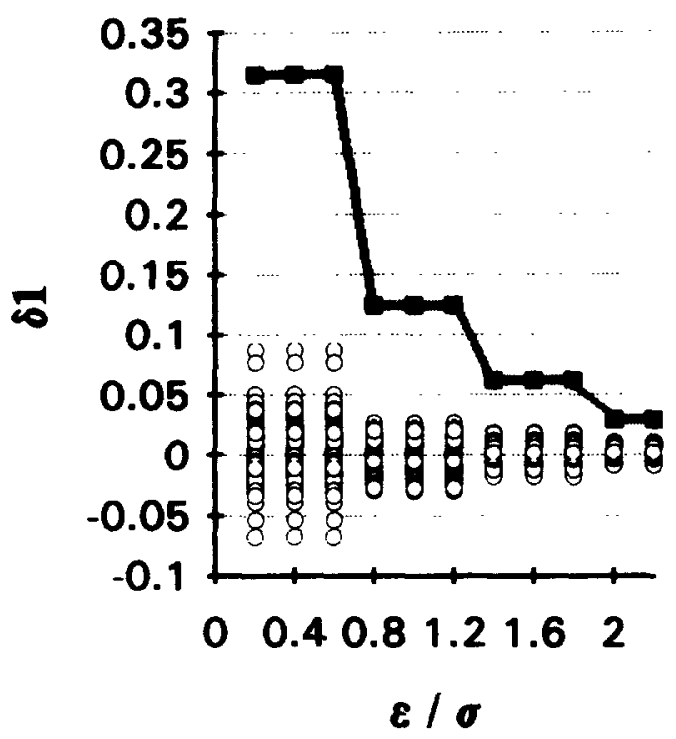

Fig. 10. $\delta_{1}$ as a function of $\varepsilon / \sigma$, with $\sigma=5.11$ days, for the rate of seizures data $y(n)$ per 2 days for patient III (solid line) and the shuffled versions of $y(n)$ (scattered points). The $\delta_{1}$ is clearly statistically significant.

$\bar{y}=1.00$ seizure per 2 days, and following the same logic as in the previous cases, we see that the detected dependence in the rate of seizures data $y(n)$ is also consistent with the $\left(\delta_{1}, \tau=1\right)$ dependence implied by the analysis of the interseizure intervals $x(n)$ of this patient as shown in Table 3.

For patient IV, even longer seizure rate dependencies are observed in $y(n)$; with $T=1$ week, $\left(\delta_{1}\right.$, $\tau=1)$ and $\left(\delta_{3}, \tau=1\right)$ are the most statistically significant pairs. Since this patient had a mean of 1.57 seizures per week, $\left(\delta_{3}, \tau=1\right)$ suggests an interdependence over five seizures, which again is in qualitative agreement with the significant pairs $\left(\delta_{1}, \tau=5\right)$ and $\left(\delta_{2}, \tau=3\right)$ from the interseizure data.

For patient $\mathrm{V}$, the values of $\delta$ 's are all small and not strongly statistically significant except for marginal dependence for $\left(\delta_{2}, \tau=1\right)$. The existing evidence for this patient's seizures is that they originate from a cortical lesion caused by trauma. The epileptogenic focus is not in the temporal lobe, as in the rest of the patients, but in the occipital lobe. Hence, anatomical differences may have had an important impact upon the low level of detected dependencies.

\section{Discussion}

This study indicates that partial complex seizures are not Poisson or independent and identically distributed (IID) in time. The Poisson assumption was not valid in any of our patient data sets. The presence of time dependencies in our data indicates that the occurrence of seizures cannot be explained solely on the basis of an IID random process. The seizure occurrences exhibited strong nonrandom structures in each of the five patients we examined. In patients with seizures of mesial temporal lobe origin (four out of five patients) the patterns of time dependencies were qualitatively similar. In the fifth patient, with seizures of occipital lobe origin, the nonrandom structure was qualitatively different than in the other four patients.

Time dependencies were detected in the seizure interval analysis as well as in the analysis of seizure rate. The exact pattern of time dependencies was unique to each patient. For each case, time dependencies in the seizure rate data were seen more clearly, that is, with a higher level of statistical significance than in the seizure interval data (e.g., compare Fig. 5(a) with Fig. 9(a)). A possible explanation of this observation is that it is the seizure rate that is slowly modulated over time. Such slow modulation could result from intrinsic or extrinsic factors such as changing concentrations of anticonvulsant drugs or hormonal variations. Modulation of the neuronal generator within a homeostatic environment is also a possibility. The effects of some of these factors on seizure recurrences have been reported in the literature [1-3].

$\Lambda$ Poisson process as an explanation for the seizure occurrences was rejected by our study as well as by that of Balish and co-workers [1]. However, it cannot be assumed that the time dependencies found in our patients would be detected by the quasi-likelihood model they employed. The SavitGreen method we applied is sensitive to both linear and nonlincar time dependencics and is a model free procedure. Most of the detected time dependencies are statistically significant even with an uncertainty in the recording of a seizure equal to one third of the standard deviation between sei- 
zures.

The results of our study as well as those of Balish and co-workers [1] appear to contradict the findings of Milton and co-workers [23]. Milton et al., using traditional statistical methods, found no evidence for nonrandom seizure occurrences in most of their patients. The occurrences were consistent with a homogeneous Poisson process in approximately $50 \%$ of their patients. There are several possible explanations for the discrepancies among the studies. For example, Milton's group included patients with both generalized and partial seizures. The study by Balish et al., like the present study, included only patients with partial seizures. Since the underlying mechanism and pathophysiology differ for generalized and partial epilepsy, it is reasonable to postulate that these different mechanisms generate seizures with different temporal patterns. This idea is supported by the observation in our study that the seizures in patient $\mathrm{V}$ demonstrated much weaker short-term structure than the rest of our patients. The seizures in this patient originated from the occipital lobe, whereas in the rest of the patients from the temporal lobe.

A second factor that may have contributed to the differences among the studies is that Milton's group analyzed 5-76 seizures, Balish's group 30576 seizures and our group 668-1147 seizures per patient. Analysis of short data segments weakens the statistical significance of deviations from a random distribution, especially when undersampling, nonstationarities or long-term dependencies exist in the data. Finally, each of these studies employed different statistical methods. In Milton's study classical statistical methods were employed. These methods assume a constant mean seizure rate over the time period of observation. However, the period of observation per patient was too short to support such an assumption. They also pointed out that their results could not exclude the possibility of a nonlinear deterministic process generating similar patterns to the ones they found in their patients. On the other hand, the statistical methods in the Balish's study involved fitting the data with a predetermined deterministic semi-linear model. Their approach might miss any dependence that differs from the ones that are included in its structure. The statistical methods employed in this study are sensitive to both linear and nonlinear dependencies in a data series. The method does require a relatively large amount of data to reveal accurately the underlying dependencies. For a simple interpretation, it also assumes stationarity of the data within the analyzed segment. This stationarity assumption is shared by almost every nonparametric statistical approach in time series analysis.

This study has implications for the selection of mathematical models for the study of seizure occurrences in epileptic patients. Clearly, the use of linear models has limited application and could result in erroneous conclusions. The AR model is a popular model for analyzing linear systems. The limitations of the AR model were demonstrated in this study. For example, it was not possible through AR modeling to detect known time dependencies in the data segments generated by the nonlinear logistic equation. Given the limitations of our understanding of the mechanisms underlying the generation and timing of seizures, specific model fitting in occurrences of seizures may be premature. Although it is not necessary to employ any model in order to further understand the dynamical processes underlying complex nonlinear processes $[21,24,29]$, using the dependencies revealed by our analysis one could define the time lags that should be included in a prospective nonlinear model.

By having analyzed the interseizure intervals and extracted hidden dependencies among the intervals, one may be able to predict the occurrence of the next seizure within a tolerance, $\varepsilon$. Given the conditional probabilistic nature of the statistics of the method we propose for the analysis of the scizure series data, it should be possible to develop a predictive algorithm of upcoming seizures given sufficient knowledge of the previous seizures. The ability to predict seizures has important therapeutic implications. For example, it may be possible to modify pharmacotherapy to provide more protection during time intervals when seizures are more likely to occur.

The prospect that seizures occur in a probabilistically deterministic nonlinear fashion also has implications for the design of clinical trials of anti- 
epileptic drugs. Currently, statistical analysis of clinical trial data assumes that seizures occur randomly in any given time interval. The results of this study indicate that this assumption is false. Thus, alternative statistical methods to evaluate drug efficacy should be considered. We believe that the information provided by the $\delta$ profiles will eventually be useful in the construction of pertinent schemas for the evaluation of drugs during experimental treatments of epileptic patients, as well as a better characterization and understanding of the mechanism of generation and timing of epileptic seizures.

\section{References}

[1] Balish. M.. Albert, P.S. and Theodore, W.H., Seizure frequency in intractable partial epilepsy: a statistical analysis, Epilepsia, 32 (1991) 642-649.

[2] Binnie, C.D., Narts, J.H.P. and Houtkooper, M.A., Temporal characteristics of seizures and epileptiform discharges, Electroenceph. Clin. Neurophysiol., 58 (1984) 498-505.

[3] Bowman, T., Leppik, I. and Haus, E., Periodicity of seizure activity in persons with complex partial seizures, Epilepsia, 25 (1984) 658.

[4] Box, G.E.P. and Jenkins, G.M., Time Series Analysis, Forecasting and Control, Holden-Day, San Francisco, CA, 1970.

[5] Burg, J.P., The relationship between maximum entropy and maximum likelihood spectra, Geophysics, 37 (1972) 375-376.

[6] Campbell, D.K.. Nonlinear science: from paradigms to practicalities, Los Alamos Sci., 15 (1987) 218-260

[7] Devaney, R.L., An Introduction to Chaotic Dinamical Systems, Addison-Wesley, New York, NY. 1986.

[8] Diaconis. P., Graham, R.L. and Cantor. W.M., The mathematics of perfect shufles, Adv. Appl. Math., 4(1983) 175-196.

[9] Efron, B. and Tibshirani, R., Bootstrapping, Stat. Sci., I (1966) $54-77$.

[10] Feigenbaum, $M, J$., Universal behavior in nonlinear systems, Physica D, 7 (1983) 16-39.

[11] Feller, W., An Introduction to Probability Theory and Its Applications, Vol. 1, 2nd edn., Wiley, New York, NY. 1962.

[12] Grassberger, P. and Procaccia, 1, Measuring the strangeness of strange attractors, Physica 0.9 (1983) 189-208.

[13] Grassberger, P.. Information flow and maximum entropy measures for 1-D maps, Physica D, 14 (1985) 365-373

[14] Green, M. and Savit, R., Dependent variables in broad band continuous time series, Physica D. 50 (1991) $521-544$.
[15] Hopkins, A., Davies, P. and Dobson, C., Mathematical models of patterns of seizures; their use in the evaluation of drugs. Arch. Neurol., 42 (1985) 463-467.

[16] lasemidis, L.D., Sackellares, J.C., Zaveri. H.P. and Williams, W.J., Phase space topography of the electrocorticogram and the Lyapunov exponent in partial seizures, Brain Topogr., 2 (1990) 187-201.

[17] Iasemidis, L.D. and Sackellares, J.C.. The temporal evolution of the largest Lyapunov exponent on the human epileptic cortex. In: D.W. Duke and W.S. Pritchard (Eds.), Measuring Chaos in the Human Brain, World Scientific. Singapore, 1991, pp. 49-82.

[18] lasemidis, L.D., Sackellares, J.C. and Savit, R.S., Quantification of hidden time dependencies in the EEG within the framework of nonlinear dynamics. In: B.H. Jansen and M.E. Brandt (Eds.), Nonlinear Dynamical Analysis of the EEG, World Scientific, Singapore, 1993, pp. $30-47$.

[19] Jensen, R.V.. Classical chaos, Am. Sci., 75 (1987) 168-171.

[20] Kay, S.M. and Marple, S.L., Spectrum analysis at modern perspective, Proc. IEEE, 69 (1981) 1380-1419.

[21] Mayer-Kress, G. (Ed.), Dimension and Entropies in Chaotic Systems, Springer-Verlag. New York, NY, 1986.

[22] Makhoul, J., Linear prediction: a tutorial review, Proc. IEEE, 63 (1975) 561-580.

[23] Milton, J.G., Gotman, J., Remillard, G.M. and Ander. mann. F., Timing of seizure recurrence in adult epileptic patients: a statistical analysis, Epilepsia, 28 (1987) $471-478$.

[24] Oppenheim, A.V., Signal processing in the context of chaotic signals. IEEE Int. Conf, ASSP, 4 (1992) 117-120.

[25] Papoulis, A.. Probability, Random Variables and Stochastio Processes, 2nd edn., McGraw-Hill, New York, NY, 1984.

[26] Parker. T.S. and Chua. L.O., Chaos: A tutorial for engineers, Proc. IEEE. 75 (1987) 982-1008.

[27] Proakis, J.G. and Manolakis, D.G., Introduction to Digital Signal Processing, Macmillan, London, 1988.

[28] Savit, R. and Green, M.. Time series and dependent variables, Physica D, 50(1991) $95-116$.

[29] Sidorowich. J.J.4 Modeling of chaotic time series for prediction, interpolation and smoothing, IEEE Int. Conf. ASSP. 4 (1992) $121 \cdot 124$

[30] Snyder, D.L., Random Point Processes, Wiley. New York, NY, 1975

[31] Swinney. H.L... Observations of order and chaos in nonlinear systems, Phwsict $D, 7$ (1983) $\} 15$

[32] Takens, $F_{\text {., }}$ Detecting strange attractors in turbulence. In: D.A. Rand and L.S. Young (Eds.), Lecture Notes on Mathematics, Vol. 898, Springer-Verlag, 1980, pp. 366 381.

[33] Theiler, J., Eubank, S., Longtin. A., Galdrikian. B. and Farmer, J.D., Testing for nonlinearity in time series: the method of surrogate data, Physica D, 58 (1992) 7794

[34] Whitney, H.. Differentiable manifolds, Ann. Math., 37 (1936) $645-680$

[35] Wu, K., Savit, R. and Brock, W., Statistical tests for deterministic effects in broad band time series, Physica $D$. in press. 